\title{
Self-Assembly of Polyelectrolyte Rods in Polymer Gel and in Solution: Small-Angle Neutron Scattering Study
}

\author{
Yu. D. Zaroslov, ${ }^{\dagger}$ V. I. Gordeliy, ${ }^{\ddagger}$ A. I. Kuklin, ${ }^{\ddagger}$ A. H. Islamov ${ }^{\ddagger}$ O. E. Philippova, ${ }^{\dagger}$ \\ A. R. Khokhlov, $*, t$ and G. Wegners \\ Physics Department, Moscow State University, 117234 Moscow, Russia; Laboratory of Neutron Physics, \\ J oint Institute for Nuclear Research, 141980 Dubna, Russia; and Sol id State Chemistry Department, \\ Max-Planck-Institute for Polymer Research, D-55128 Mainz, Germany
}

Received November 19, 2001; Revised Manuscript Received February 11, 2002

\begin{abstract}
Self-aggregation of rigid-rod poly(sodium p-phenylenesulfonate) in aqueous solution and inside water-swollen polyacrylamide gel was studied by small-angle neutron scattering. It was shown that both inside the hydrogel and in solution polyelectrolyte rods self-assemble into cylindrical aggregates having eight to nine single polymer chains in the cross-section, the chains being aligned parallel to the axis of the aggregate. The length of these aggregates is much higher than the contour length of a single chain. Gels with embedded rods were studied by contrast variation method in order to examine separately the scattering by the gel and by the rods. Two important observations were made. First, it was shown that the ordering of the rods in the gel resembles that in solution. Second, it was shown that the gel itself is more homogeneous in the presence of rods. M ost probably, this effect is due to mobile counterions of rods, which counteract the formation of spatial inhomogeneities in the network during synthesis, because in an inhomogeneous network mobile counterions should be also distributed nonuniformly that is associated with significant translational entropy losses.
\end{abstract}

\section{Introduction}

Rigid-rod polyelectrolytes based on sulfonated poly(p-phenylene) are able to aggregate in aqueous media due to the hydrophobic character of their backbone. This aggregation does not lead to precipitation because the charged sulfonate groups ensure the solubility of the aggregates in water. The first data on self-aggregation of sulfonated poly(p-phenylene)s in aqueous solutions were obtained by SAXS. ${ }^{1}$ SAXS data showed that aggregated polymer chains take the form of columnar micelles, in which the phenylene backbones are oriented parallel to the axis of the micelle. ${ }^{1}$ The magnitude of the radial aggregation number of the micelles was estimated for a series of sulfonated poly(p-phenylene) samples differing in charge density and in hydrophobicity. No information was obtained about the length of these aggregates from the evaluation of the SAXS data. It was emphasized ${ }^{1}$ that the observed behavior of these polymers in solutions resembles the formation of supermolecular structures of some biogenic wormlike or stiff polyelectrolytes such as DNA or collagen. Therefore, a detailed study of the aggregates of rods in water could lead to better understanding of the processes of selforganization of biological macromolecules as well.

On the other hand, it was shown that the ability of the macromolecules to aggregate can be used to immobilize them inside a water-swollen gel without formation of covalent bonds with the network. ${ }^{2}$ The incorporation of rodlike polyelectrolyte in superabsorbent hydrogels was proposed as a simple and efficient way for the improvement of mechanical properties of hydrogels in the swollen state which could be important for practical applications. ${ }^{2}$ To control the properties of

\footnotetext{
* Corresponding author. E-mail: zaroslov@polly.phys.msu.su. $\dagger$ Moscow State University.

₹ J oint Institute for Nuclear Research.

$\S$ Max-Planck-Institute for Polymer Research.
}

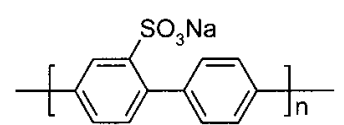

Figure 1. Chemical structure of poly(sodium p-phenylene sulfonate).

the gels with embedded rigid rods, the self-assembly of rod macromolecules inside the network should be studied. In our previous communication, ${ }^{2}$ an indirect evidence of the self-aggregation of rods inside the gel was obtained from the results of the experiments on the swelling behavior of the gels. The aim of this work is to investigate the self-assembly of rigid-rod polyelectrolyte inside the water-swollen gel and to establish effects of the gel on the self-assembly of rods as well as of the rods on the gel structure.

\section{Experimental Section}

Materials. The synthesis of poly(sodium p-phenylene sulfonate) PPP2 (Figure 1) is described el sewhere. ${ }^{3,4}$ Characterization of the precursor polymer which had sulfonate groups protected as 3,5-di-tert-butylphenolate is presented in refs 3 and 4 . The degree of polymerization of the precursor polymer is equal to $P_{n}=23.5$; the contour length equals $18 \mathrm{~nm}$. The persistence length of the precursor polymer was shown to be equal to $13 \mathrm{~nm}$. This value can be regarded as the lower bound of the persistence length of the polyel ectrolyte under study. Therefore, the PPP2 chains can be considered as rodlike chains.

Polymer solutions were prepared by weighing the polymer and the solvent $\left(D_{2} O\right)$, mixing them and stirring at a temperature not higher than $95^{\circ} \mathrm{C}$ for $20 \mathrm{~min}$.

For preparation of the polyacrylamide (PAAm) gels with embedded PPP 2 rods, the mixture of $\mathrm{D}_{2} \mathrm{O}$ and $\mathrm{H}_{2} \mathrm{O}(80 / 20 \mathrm{v} / \mathrm{v})$ of given composition was used as a solvent. The $\mathrm{D}_{2} \mathrm{O} / \mathrm{H}_{2} \mathrm{O}$ mixture was added to $90 \mathrm{mg}$ of acrylamide and to a calculated amount of PPP2 to have a resulting mass of the sample of 850 $\mathrm{mg}$. Then $50 \mathrm{mg}$ of $0.126 \mathrm{~mol} / \mathrm{L} \mathrm{N}, \mathrm{N}^{\prime}$-methylenebis(acrylamide) solution prepared in the same solvent was added, and the mixture was stirred at room temperature during at least 


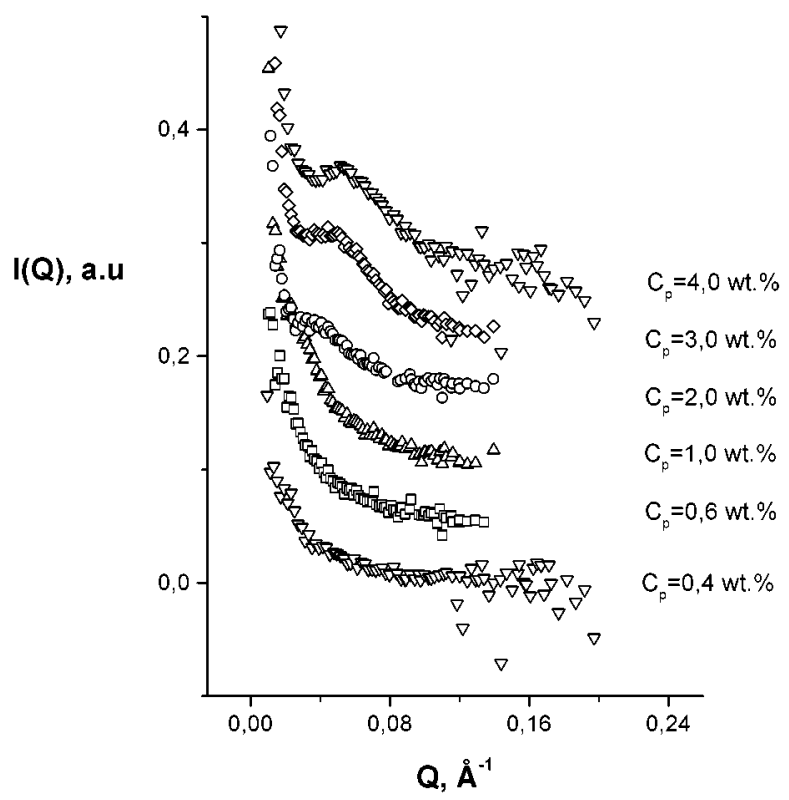

Figure 2. Variation of SANS intensity with polymer concentration $\mathrm{C}_{\mathrm{p}}$ in $\mathrm{D}_{2} \mathrm{O}$; the values of $\mathrm{C}_{\mathrm{p}}$ are indicated in the figure. Starting from the lower curve $\left(C_{p}=0.4\right.$ wt \%), each curve is displaced with respect to the preceding one by 0.05 units along the $y$-axis in order to avoid overlap.

$12 \mathrm{~h}$. After the mixture was stirred, $50 \mathrm{mg}$ of solution of ammonium peroxodisulfate $\left(4.4 \times 10^{-4} \mathrm{~mol} / \mathrm{L}\right)$ and $50 \mathrm{mg}$ of a solution of $\mathrm{N}, \mathrm{N}, \mathrm{N}^{\prime}, \mathrm{N}^{\prime}$-tetramethylethyl enediamine $\left(4.4 \times 10^{-4}\right.$ $\mathrm{mol} / \mathrm{L}$ ) prepared in the same $\mathrm{D}_{2} \mathrm{O} / \mathrm{H}_{2} \mathrm{O}$ mixture were added. The mixture was stirred again for $5 \mathrm{~min}$ and poured into special dismountable cell for further polymerization, which was performed over at least $24 \mathrm{~h}$.

$\mathrm{D}_{2} \mathrm{O}(99.9 \%)$ from Fluka was used as received. Water was purified with a Milli-Q system (Millipore).

Small-Angle Neutron Scattering. SANS measurements were done at the YuMO instrument of the high-flux pulsed reactor IBR-2 at Frank Laboratory of Neutron Physics, J oint Institute for Nuclear Research, Dubna, Russia. ${ }^{5}$

The measurements of solutions were performed in standard quartz Helma cells, while gels were measured in specially designed dismountable cells with quartz windows and a Teflon spacer. The path length of neutron beam in both cases was equal to 1 or $2 \mathrm{~mm}$ depending on the relative content of protons in the sample.

All data were treated according to standard procedures of small-angle isotropic scattering. The spectra were corrected for transmission, sample thickness, and absolute scattering intensity. ${ }^{5}$ The mixture of solvents at the same composition and temperature was used as background for the samples.

\section{Results and Discussion}

Before studying the self-aggregation of polyelectrolyte rods in water-swollen gels, let us consider a simpler system, which will be used further for comparison, namely polyelectrolyte rods in aqueous solution.

A. Polyelectrolyte Rods in Solution. (a) E ffect of Polymer Concentration. The neutron scattering intensity I depending on the scattering vector $\mathrm{Q}$ for solutions of PPP2 of different concentrations in $\mathrm{D}_{2} \mathrm{O}$ is shown in Figure 2. It is seen that at polymer concentrations lower than 1 wt \% I (Q) is a monotonically decreasing function with Q. However, above this concentration, a correlation (diffraction) peak is clearly visible. The position of the peak is shifted to higher $\mathrm{Q}$ values (i.e., to smaller spacing) with the increase of polymer concentration.

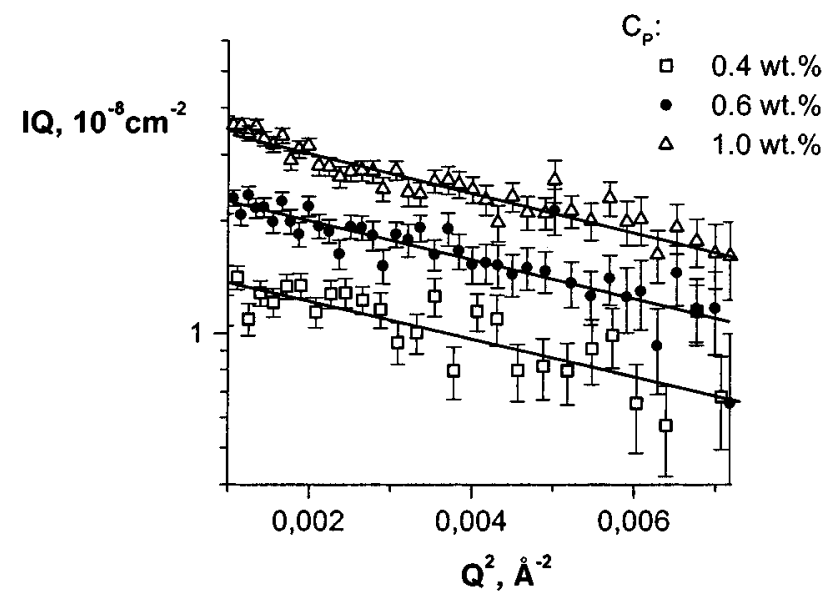

Figure 3. SANS intensities I plotted as IQ vs $\mathrm{Q}^{2}$ for solutions of PPP2 in $\mathrm{D}_{2} \mathrm{O}$ at low polymer concentrations $\left(C_{p}=0.4,0.6\right.$, and $1.0 \mathrm{wt} \%)$.

In general, the scattering intensity I(Q) of a system of particles is described by

$$
\mathrm{I}(\mathrm{Q})=|\mathrm{F}(\mathrm{Q})|^{2} \mathrm{~S}(\mathrm{Q})
$$

where $\mathrm{Q}=4 \pi(\sin \theta) / \lambda$ is the scattering vector ( $\theta$ is a half of scattering angle, and $\lambda$ is the neutron wavelength), $F(Q)$ is the form factor of the particle and $S(Q)$ is the structure factor. When there is no correlation in the positions of the particles or when the magnitude of Q corresponds to a much lower length scale than the interparticle distance, the scattering can be regarded as the scattering of independent particles, i.e.

$$
\mathrm{I}(\mathrm{Q})=\mathrm{n}_{\mathrm{p}}|\mathrm{F}(\mathrm{Q})|^{2}
$$

where $n_{p}$ is the number of the particles.

Let us first analyze the scattering curves for polymer solutions at $C_{p}=0.4$ and 0.6 wt $\%$, which do not contain a diffraction peak. Such analysis can be applied also to the wide-angle part of scattering curve which is not "disturbed" by the diffraction peak in the case of polymer solutions of higher concentration (1.0 wt \%). The scattering spectra obtained for the samples with $C_{p}=0.4$, 0.6 , and 1.0 wt \% are presented in the form of $\log (\mathrm{IQ})$ vs $\mathrm{Q}^{2}$ plots in Figure 3 . It is seen that in these coordinates the scattering curves are straight lines, which is typical for the scattering of rodlike particles. Therefore, we can conclude that the aggregates of PPP2 in aqueous solution have the form of long rods, which seems reasonable taking into account the rigid character of poly(p-phenylene) backbone itself.

In the case of the scattering of rodlike particles, when one dimension $L$ (length) is much higher than the others, the form factor of the particle can be expressed as 6

$$
|F(Q)|^{2}=I_{L}(Q) I_{C}(Q)
$$

where the function $I_{L}(Q)$ is proportional to the length of the particle $\mathrm{L}: \mathrm{I}_{\mathrm{L}}(\mathrm{Q})=\pi \mathrm{L} \mathrm{Q}^{-1}$, while the function $I_{c}(Q)$ does not depend on the length of the rod, but is determined by the cross-sectional structure of the rods. In the range of scattering vectors $1 / L<Q<1 / R_{C}$ 
Table 1. Summary of the SANS Data for Solutions of $P P P 2$ in $D_{2} O$

\begin{tabular}{cccccc}
\hline $\mathrm{C}_{\mathrm{p}}$, wt \% & $\mathrm{R}_{\mathrm{c},} \AA$ & $\begin{array}{c}\mathrm{IQ} \times 10^{3} \mathrm{~cm}^{-2} \\
(\mathrm{Q}=0)\end{array}$ & $\mathrm{Q} *, \AA^{-1}$ & $\mathrm{~d}_{\mathrm{b}}, \AA$ & $\mathrm{a}_{\mathrm{rad}}$ \\
\hline 0.4 & $15.8 \pm 1.4$ & $1.6 \pm 0.05$ & & & \\
0.6 & $15.8 \pm 0.4$ & $2.6 \pm 0.1$ & & & \\
1.0 & $14.0 \pm 0.8$ & $3.67 \pm 0.07$ & 0.034 & 185 & 8.8 \\
2.0 & & & 0.047 & 134 & 9.3 \\
3.0 & & & 0.058 & 108 & 9.0 \\
4.0 & & & 0.066 & 95 & 9.3
\end{tabular}

this function is approximately equal to

$$
I_{c}(Q)=I_{c}(0) \exp \left[-\frac{R_{c}{ }^{2} Q^{2}}{2}\right]
$$

where $R_{c}$ is the radius of gyration of the cross section and $I_{C}(0)$ is the scattering intensity at zero scattering vector

$$
\mathrm{I}_{\mathrm{c}}(0)=\bar{\rho}_{\mathrm{c}}{ }^{2} \mathrm{~S}_{\mathrm{c}}^{2}
$$

where $\bar{\rho}_{c}$ is the average neutron scattering density of the cross-section, $\mathrm{S}_{\mathrm{c}}$ is the area of the cross-section.

Therefore, from the slope of linear dependence of log(IQ) vs $\mathrm{Q}^{2}$ the radius of gyration of the cross-section $\mathrm{R}_{\mathrm{c}}$ can be determined, according to the eqs 3 and 4 . The obtained values of $R_{c}$ are presented in Table 1 . It is seen that they are equal to ca. $15 \AA$ independently of the concentration of the polymer. Note that this value is much higher than the estimated radius of cross section of a single macromolecule (ca. $5 \AA$ ).

The analysis of the experimental data thus obtained allows us also to estimate the length $L$ of the scattering rods. The length of the particles is given by the following asymptotic relation: ${ }^{6}$

$$
\mathrm{L}=\pi \mathrm{l}(0) /[\mathrm{I}(\mathrm{Q}) \mathrm{Q}]_{\mathrm{Q}=0}
$$

The value of $[\mathrm{I}(\mathrm{Q}) \mathrm{Q}]_{\mathrm{Q}=0}$ was obtained by extrapolation of the straight line of the dependence $\log (\mathrm{IQ})$ vs $\mathrm{Q}^{2}$ to zero scattering vector $\mathrm{Q}=0$ (Table 1 ). As to the value of I (0), we used as approximation the scattering intensity at the lowest measured scattering vector $\left(Q_{\min } \approx\right.$ 0.01 ). The use of this value instead of I (0) in eq 5 will give us the lower bound of the length $L$ of the rods. The result is that the length of the rods must be larger than $36 \mathrm{~nm}$, which far exceeds the average contour length of the individual polymer chains (18 nm). Therefore, PPP2 macromolecules in aqueous solution form rodlike aggregates, the radius and length of which are much larger than the corresponding parameters for the constituting macromolecules.

A scattering maximum appears with increasing polymer concentration as it is seen from Figure 2. It can be due to a correlation hole effect around highly charged polyelectrolyte chains promoted by electrostatic repulsion. ${ }^{7}$ To determine accurately the positions of the maxima of the diffraction peaks it is necessary to separate the structure factor $\mathrm{S}(\mathrm{Q})$ from the form factor $F(Q)$. The form factor $|F(Q)|^{2}$ was determined by fitting the experimental data for the samples with $0.4,0.6$, and 1.0 wt \% of polymer by eq 2 combined with eqs 3 and 4 (F igure 3). Then the structure factor S(Q) was obtained by dividing the scattering intensity $\mathrm{I}(\mathrm{Q})$ by the form factor $|F(Q)|^{2}$ determined by eq 1 . F or the samples with

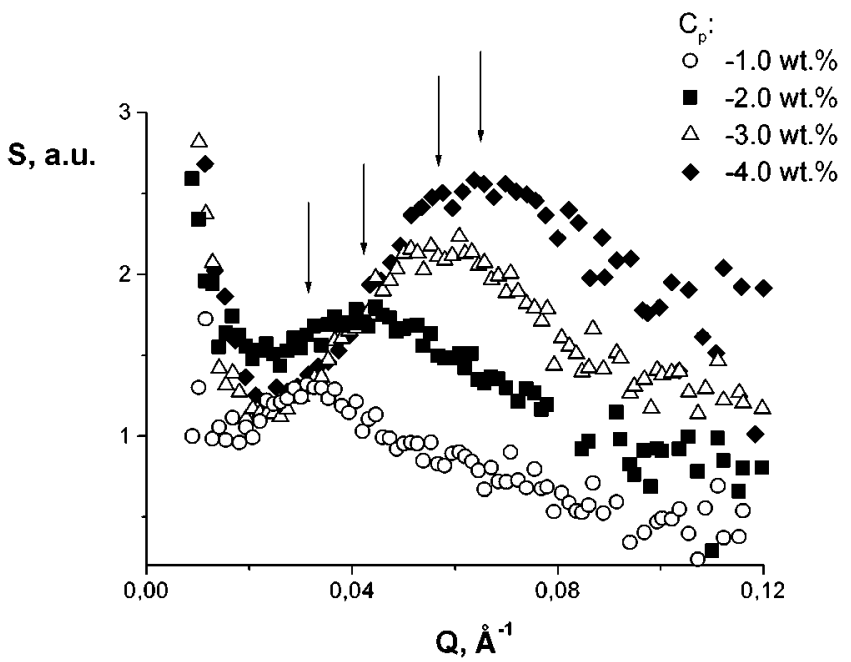

Figure 4. Variation of the structure factor with polymer concentration $C_{p}$ in $D_{2} O$ solution; the values of $C_{p}$ are indicated in the figure. Positions of structure peaks are marked by arrows.

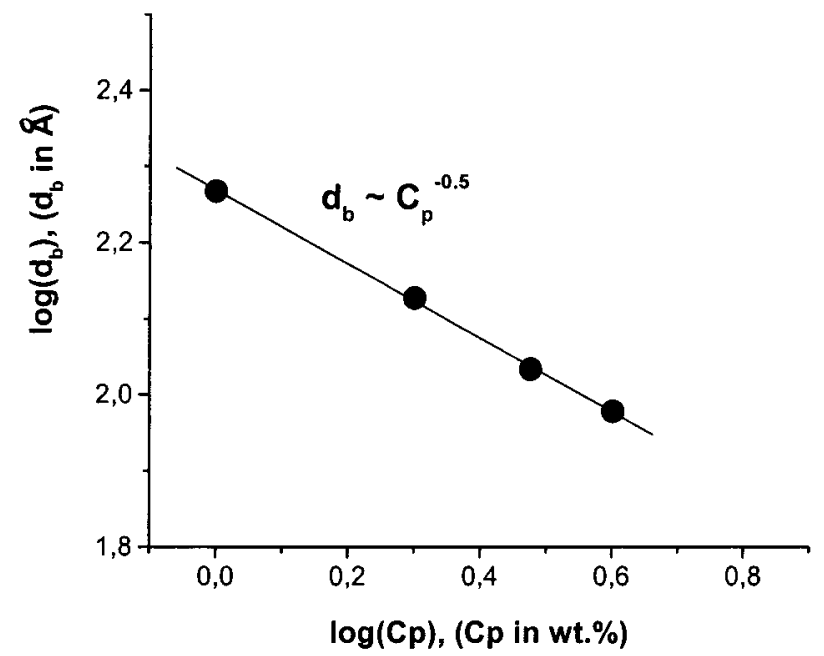

Figure 5. Variation of the average interaggregate distance $d_{b}$ with polymer concentration $C_{p}$ in $D_{2} \mathrm{O}$ solution. Solid line corresponds to the slope -0.5 .

higher polymer concentrations ( 3 and 4 wt \%) another approach was used to estimate the structure factor. Since eq 4 is valid only at $Q<1 / R_{c}$, it cannot be applied to the samples of 3.0 and 4.0 wt \% of PPP2. For these samples, more general equation for the form factor of rodlike scattering particles $\left(\mathrm{F}(\mathrm{Q}) \sim \mathrm{Q}^{-1}\right)$ can be used in order to estimate the magnitude of the structure factor. The results are shown in Figure 4.

The values of Q* obtained at the diffraction maxima are compiled in Table 1. It is seen that the peak position moves toward higher $\mathrm{Q}$ values with increasing polymer concentration. If we regard the position of the peak as a measure of the most probable distance $d_{b}$ between the rodlike aggregates, we can calculate the mean interaggregate distance $d_{b}=2 \pi / Q^{*}$. The values of $d_{b}$ thus obtained are presented in Table 1. It is seen that they vary from 185 to $95 \AA$ as the polymer concentration increases from 1.0 to $4.0 \mathrm{wt} \%$. The mean interaggregate distances scale with polymer concentration according to the power law $d_{b} \sim C_{p}{ }^{-0.5}$ (Figure 5). This can be regarded as an indication of the two-dimensional structure formed by rodlike aggregates in the plane normal to the axis of the aggergates. ${ }^{7,8}$ 

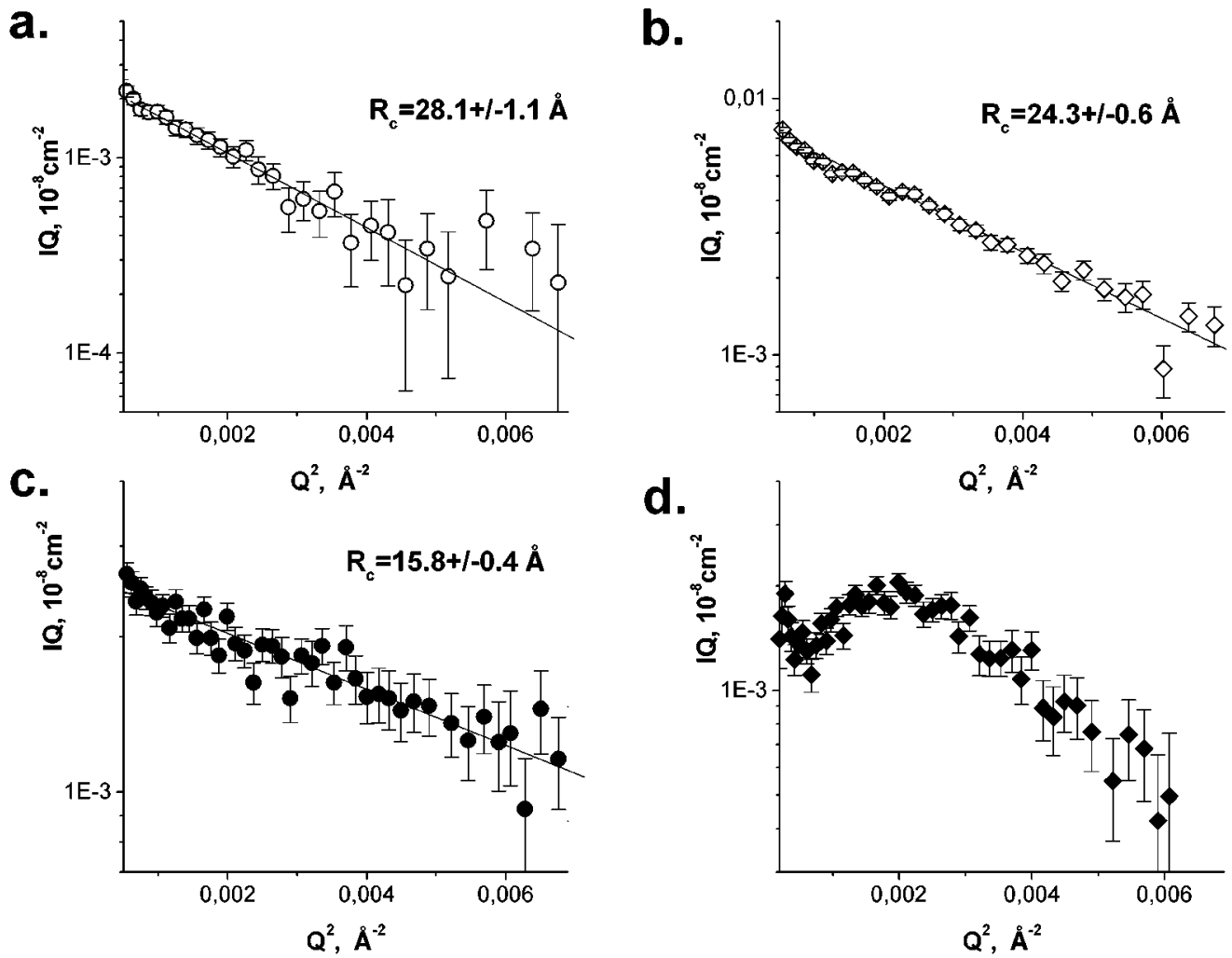

Figure 6. Effect of salt on the SANS intensity of PPP2 solutions in $\mathrm{D}_{2} \mathrm{O}$ at polymer concentrations 0.6 (a) and 2.0 wt \% (b). Concentration of $\mathrm{NaCl}$ is $0.2 \mathrm{~mol} / \mathrm{L}$. For comparison spectra of polymer solutions without salt are also presented (0.6 (c) and 2.0 wt \% (d)).

As was mentioned above, the radius of gyration of the cross section of a rod is higher than the cross section of a single polymer chain. Therefore, the rodlike particles observed by SANS can be considered as aggregates consisting of several polymer chains arranged parallel to each other. The position of the maximum of the peaks Q* allows one to calculate the radial aggregation number $a_{\text {rad }}$, i.e., an average number of polymer chains in the cross section of the rodlike aggregate. Assuming hexagonal lattice of the packing of the rods ${ }^{1}$ one can calculate $a_{\text {rad }}$ as follows

$$
a_{r a d}=\frac{2}{\sqrt{3}} d_{b}^{2} c_{A}
$$

where $d_{b}$ is the Bragg spacing $\left(d_{b}=2 \pi / Q *\right), c_{A}$ is the number of chains crossing a unit area perpendicular to the direction of the chains. The value of $c_{A}$ is rel ated to the weight per volume concentration $\mathrm{c}$ (in $\mathrm{g} / \mathrm{cm}^{3}$ ) of polymer by

$$
\mathrm{c}_{\mathrm{A}}=\frac{\mathrm{CN}_{\mathrm{A}} \mathrm{I}_{\text {rep }}}{\mathrm{M}_{\text {rep }}}
$$

where $\mathrm{N}_{\mathrm{A}}$ is Avogadro's number and $\mathrm{I}_{\text {rep }}$ and $\mathrm{M}_{\text {rep }}$ are the length and molecular weight of the repeat unit of polymer, respectively. The calculated values of radial aggregation number are presented in Table 1 . I t is seen that the value of $\mathrm{a}_{\mathrm{rad}}$ is constant in the studied range of polymer concentrations (1-4 wt \%) and it approximately equals 9 (Table 1 ).

An interesting experimental result should be mentioned here. Assuming homogeneous distribution of the polymer chains in the cross section of the rods, one can calculate the area $\mathrm{S}_{\mathrm{c}}$ of the cross-section by the following equation

$$
\mathrm{S}_{\mathrm{c}}=\pi\left(\sqrt{ } 2 \mathrm{R}_{\mathrm{c}}\right)^{2}
$$

Taking the experimentally determined values of $R_{c}=$ $15 \AA$ and $a_{\text {rad }}=9$ and the calculated effective crosssection area of a single molecule $A_{p} \cong 70 \AA^{2}$ one can estimate the ratio of the area occupied by the polymer to the total area of the cross section of the rod: $a_{r a d} A_{p} /$ $\mathrm{S}_{\mathrm{c}}=11 \%$. Therefore, the polymer itself occupies only $11 \%$ of the cross-section area. This implies a very loose packing of the macromolecules in the aggregates or an irregular shape of the aggregates.

Therefore, the analysis of the SANS data for PPP2 solutions in $\mathrm{D}_{2} \mathrm{O}$ shows that polyelectrolyte chains are self-assembled in rodlike aggregates, consisting of ca. 9 polymer chains arranged parallel to each other, the length of the aggregate being much longer than the length of a single polymer chain.

(b) Effect of Salt. The addition of low molecular weight salt would supposedly screen the electrostatic interactions and should favor the aggregation of similarly charged polymer chains. To check this suggestion, the SANS spectra of PPP 2 in $0.2 \mathrm{~mol} / \mathrm{L} \mathrm{NaCl}$ solutions were obtained. They are presented in Figure 6, parts a and $\mathrm{b}$. I $\mathrm{n}$ the same figure the spectra of PPP 2 solutions in pure $\mathrm{D}_{2} \mathrm{O}$ are shown for comparison as well (Figure 6 , parts $\mathrm{c}$ and d). It is seen that in the presence of $\mathrm{NaCl}$, when the electrostatic interactions shoud be effectively screened, the diffraction peak in the spectrum of $2 \mathrm{wt}$ $\%$ solution of PPP2 disappears. These data contribute to the suggestion that the spatial arrangement of the aggregates is governed by electrostatic interactions. When the electrostatic repulsion is screened by salt, the spatial correlation between aggregates disappears. 
a.

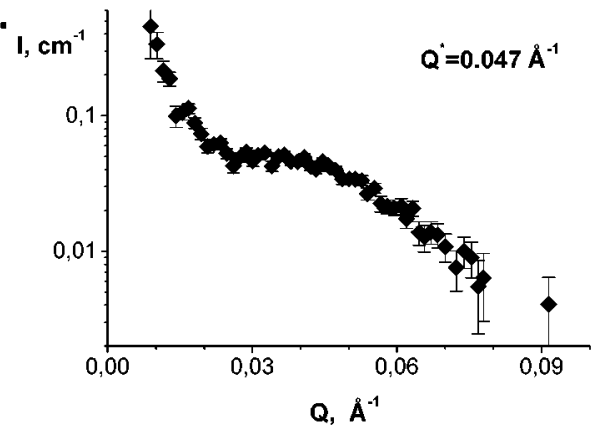

b.

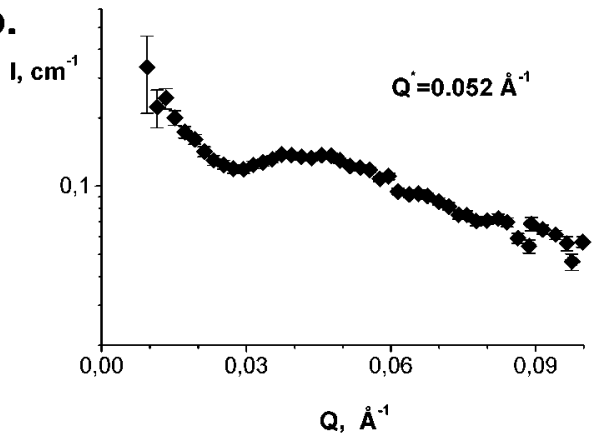

Figure 7. SANS intensity for PPP 2 inside PAAm networks (b) and in $\mathrm{D}_{2} \mathrm{O}$ solution (a). Concentration of polymer in both cases is 2.0 wt \%.

The analysis of the spectra of salt solutions of PPP2 shows that they can be presented as straight lines in coordinates of $\log (\mathrm{IQ})$ vs $\mathrm{Q}^{2}$ (Figure $6 \mathrm{a}$ and $\mathrm{b}$ ). Therefore, the aggregates in the presence of salt have a rodlike form as in pure $\mathrm{D}_{2} \mathrm{O}$. But the values of the radius of gyration in salt solution are 2-2.5-fold higher than in pure $\mathrm{D}_{2} \mathrm{O}$. Also, it was found that $\mathrm{NaCl}$ induces an increase of the scattering intensity extrapolated to zero angle, that is an increase of the length of aggregates.

Therefore, the SANS data show a significant effect of low molecular weight salt on the aggregation of rods in aqueous solution: the salt induces the growth of both the length and the radius of rodlike aggregates. The effect of salt is due to the screening of electrostatic repulsion between similarly charged polymer chains.

B. Polyelectrolyte Rods in the Gel. When studying the polyelectrolyte rods in the gel, two separate effects need consideration: the effect of the gel on the selfassembly of rods and the effect of rods on the gel structure. Let us first consider the effect of the network on the self-aggregation of polymer rods. Figure 7 shows the scattering curve for PPP2 inside a PAAm gel swollen by $\mathrm{D}_{2} \mathrm{O}$. The scattering curve for PPP 2 in $\mathrm{D}_{2} \mathrm{O}$ solution at the same concentration of polymer is presented in the same figure. It is seen that the introduction of the PPP2 macromolecules inside the gel gives rise to the appearance of the diffraction peak, the position of which being very close to that observed for aqueous solution of PPP2. Data treatment of the SANS intensity of PPP2 in the gel performed in the same way as described above allows us to estimate the mean aggregation number of the rods inside the gel. It appears to be al most the same as in the solution $\left(\mathrm{arad}_{\mathrm{rad}}=8\right)$. Therefore, assuming the rodlike structure of the PPP2 agregates inside the gel, we can conclude that their aggregation numbers are not affected by the gel.

Another important question pertains to the effect the rods may have on the structure of the gel network, as it is prepared in the aqueous solution of the rods. A

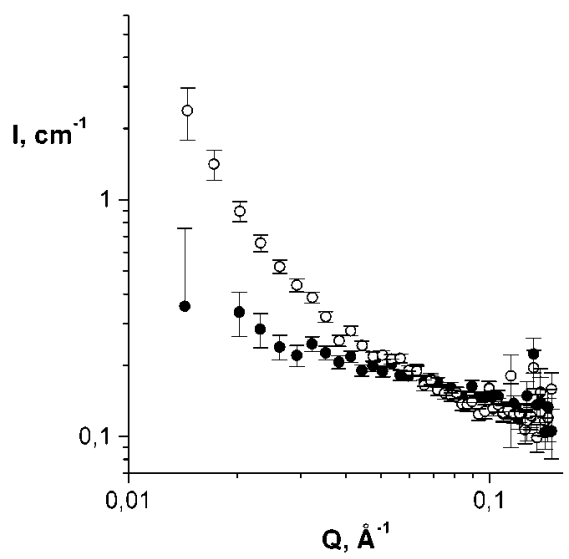

Figure 8. Scattering curves of PAAm gel with incorporated $2.0 \mathrm{wt} \%$ PPP2 (-) and without embedded pol yelectrolyte rods (O) in $\mathrm{D}_{2} \mathrm{O} / \mathrm{H}_{2} \mathrm{O}$ mixture $80 / 20 \mathrm{v} / \mathrm{v}$, matching the scattering from the rods.

contrast variation method was used to address this problem. Using values of van der Waals radii and corresponding neutron scattering lengths of the atoms of the PPP2 repeat unit it was calculated that a $\mathrm{D}_{2} \mathrm{O} /$ $\mathrm{H}_{2} \mathrm{O}$ mixture with a volume ratio of $80 / 20$ has the same scattering length density as the PPP2 molecules. The SANS intensity of the PAAm network with embedded PPP2 polymer swollen by $\mathrm{D}_{2} \mathrm{O} / \mathrm{H}_{2} \mathrm{O}$ mixture $(80 / 20 \mathrm{v} / \mathrm{v})$ matching the scattering of the polyelectrolyte rods is presented in Figure 8. The comparison with the scattering curve of PAAm gel swollen in the same $\mathrm{D}_{2} \mathrm{O} / \mathrm{H}_{2} \mathrm{O}$ mixture in the absence of the polyel ectrolyte rods shows that at low $Q$ values the scattering from the gel with rods is much smaller than the scattering of the gel without rods. Therefore, the polyelectrolyte removes long-range fluctuations within the PAAm gel structure. This result seems to be rather unexpected. Indeed, when the gel is prepared in the sol ution of rods one can expect that the distribution of the polymer density in the network should be more inhomogeneous than in the absence of rods. Nevertheless, the experimental data show the opposite effect. It may be due to the osmotic pressure of mobile counterions of rods. Indeed, since the PPP2 aggregates are bound to the polymer network, ${ }^{2}$ the fluctuations of the polymer density of the network should cause an inhomogeneous distribution of the charged rods and their free counterions, which is unfavorable because of a significant loss of the entropy of the counterions. This effect is consistent with the results of theoretical consideration made in ref 9 . On the other hand, the stabilization of the spatial arrangements of the similarly charged pol yel ectrolyte rods with some characteristic correlation length $\left(d_{b}\right)$ by electrostatic repulsion can be responsible for the effective screening of the long-range density fluctuations in polymer network. This assumption correlates well with data presented in Figure 8, where the scattering intensities of PAAm gels with and without incorporated PPP2 aggregates overlap on the scales lower than $d_{b}(Q>Q *)$. While, at smaller scattering vectors $\left(\mathrm{Q}<\mathrm{Q}^{*}\right)$, the scattering intensity in the presence of the polyel ectrolyte becomes smaller, which indicates a more homogeneous structure of the polymer network than it is in the case of the gel without rods. Therefore, not only the PAAm network affects the aggregation of rods, but also the rods influence the structure of PAAm network making it more homogeneous on a large scale. 


\section{Conclusions}

A comparative study of the aggregation of polyelectrolyte rods inside a water-swollen gel and in aqueous solutions was performed by SANS. It was shown that both in solution and in the gel the polyel ectrolyte rods form cylindrical aggregates consisting of ca. 9 single molecules per a cross section. It was demonstrated that the polyelectrolyte rods affect the structure of PAAm network making it more homogeneous.

Acknowledgment. This research was supported by RFBR Grant No. 02-03-33259 and INTAS Grant No. 00-445.

\section{References and Notes}

(1) Rulkens, R.; Wegner, G.; Thurn-Albrecht, T. Langmuir 1999, $15,4022-4025$.
(2) Philippova, O. E.; Rulkens, R.; Kovtunenko, B. I.; Abramchuk, S. S.; Khokhlov, A. R.; Wegner, G. Macromol ecules 1998, 31, 1168-1179.

(3) Rulkens, R.; Schulze, M.; Wegner, G. Macromol. Chem., Rapid Commun. 1994, 15, 669-676.

(4) Rulkens, R.; Wegner, G.; Enkelmann, V.; Schulze, M. Ber. Bunsen-Ges. Phys. Chem. 1996, 100, 707-714.

(5) Ostanevich, Yu. M. Macromol. Chem., Macromol. Symp. 1988, 15, 91-103.

(6) Svergun, D. I.; Feigin, L. A. X-ray and Neutron Small-Angle Scattering; Nauka: Moscow, 1986.

(7) De Gennes, P. G.; Pincus, P.; Velasco, R. M.; Brochard, F. J . Phys. 1976, 37, 1461-1472.

(8) Essafi, W.; Lafuma, F.; Wiliams, C. E. In Macro-I on Characterization. From DiluteSolutions to Complex Fluids; Schmitz K. S., Ed.; ACS Symposium Series 548; American Chemical Society: Washington, DC, 1994; p 278.

(9) Zeldovich, K. B.; Khokhlov, A. R. Macromolecules 1999, 32, 3488-3494.

MA0120207 